\title{
RELATO DE EXPERIÊNCIA: UTILIZAÇÃO DO PROJETO TERAPÊUTICO SINGULAR COMO ESTRATÉGIA DE PRODUÇÃO DE UM CUIDADO INTEGRAL
}

\section{EXPIRIENCE REPORT: AN APPLICATION OF THE SINGULAR THERAPEUTIC PROJECT AS A STRATEGY OF PRODUTION FOR A COMPREHENSIVE HEALTH CARE}

\author{
Joana Rosa Urbano Sousa Costa ${ }^{1}$ \\ Carolina Ávila Garcia ${ }^{2}$ \\ Erivar Moisés de Lima Júnior ${ }^{3}$
}

\begin{abstract}
RESUMO: Após a criação do SUS, iniciou-se uma mudança no modelo de visão em saúde, no qual o modelo hospitalocêntrico foi dando lugar a uma nova perspectiva, com ênfase na assistência básica e o cuidado integral em saúde. Dessa maneira, o Projeto Terapêutico Singular (PTS) surge como uma estratégia para um cuidado integrativo do usuário e comunidade, que se baseia na união de proposições de condutas terapêuticas para um indivíduo levando em consideração o ser como todo em sua singularidade. O objetivo do trabalho é discutir o uso do PTS como ferramenta de construção de um cuidado integral, por meio da experiência em uma unidade de saúde da família. A metodologia utilizada é o relato de experiência do estudante. Como resultados observou-se que para construção do PTS foi importante compreender a situação de vida do ponto de vista da paciente e de cada membro da equipe, pois assim conseguiu-se propor condutas mais adequadas para o contexto daquela paciente. Logo, conclui-se que o PTS se mostrou uma ferramenta de grande utilidade no cuidado a paciente do relato de experiência, pois trouxe uma visão integrada dela que vai além do tradicional modelo biomédico hospitalocêntrico, além de que sua implantação vem motivar a reavaliação desse modelo. Devendo, portanto, haver mais debates, reflexões e problematizações acerca do assunto por parte de todos os integrantes do sistema de saúde, para que, dessa forma, seja possível cada vez mais, melhorar a assistência à saúde no Brasil.
\end{abstract}

Palavras chave: Assistência integral à saúde. Assistência primária a saúde. Estratégia de saúde da família. Estudantes.

\footnotetext{
${ }^{1}$ Acadêmica de Medicina da Universidade Federal da Paraíba (UFPB).

${ }^{2}$ Acadêmica de Medicina da Universidade Federal da Paraíba (UFPB).

${ }^{3}$ Acadêmico de Medicina da Universidade Federal da Paraíba (UFPB).
} 
ABSTRACT: After the creation of the SUS, a change in the health vision model began, in which the hospital - centered model was giving way to a new perspective, with emphasis on basic care and comprehensive health care. Thus, the Singular Therapeutic Project (known as PTS in Brazil) emerges as a strategy for a comprehensive health care of the patient and his community, which is based on the union of propositions of therapeutic behaviors for an individual taking into consideration being as a whole in its uniqueness. The objective of this paper is to discuss the use of PTS as a tool to build comprehensive care through experience in a family health unit. The methodology used is the student experience report. As results, it was observed that for the construction of the PTS, it was important to understand the life situation from the point of view of the patient and each team member, thus being able to propose more appropriate behaviors for that patient's context. Therefore, it can be concluded that the PTS proved to be a very useful tool in the care of patients with experience reports, as it brought an integrated view of it that goes beyond the traditional hospital-centric biomedical model, and its implementation motivates the reevaluation of this model. So, there should be more debates, reflections and problematization on the subject by all members of the health system, so that, thus, it is possible to increasingly improve health care in Brazil.

Keywords: Comprehensive Health Care. Primary Health Care. Family Health Strategy. Students. 


\section{INTRODUÇÃO}

Após a criação do SUS, iniciou-se uma mudança no modelo de visão em saúde, no qual o modelo hospitalocêntrico foi dando lugar a uma nova perspectiva, com ênfase na assistência básica e o cuidado integral em saúde. Dessa maneira, foi incorporada a ideia do coletivo, juntamente com a subjetividade e a importância da participação ativa do usuário na produção de saúde. (MINAYO, 2008).

Nessa linha de raciocínio, a Estratégia de Saúde da Família foi criada pelo Ministério da Saúde (MS) com o intuito de reorganizar a atenção básica no país, através dos princípios do SUS, levando a uma expansão e consolidação da assistência básica por facilitar uma reestruturação do processo de trabalho, favorecendo assim a ampliação da resolutividade da situação de saúde das pessoas e coletividades. (BRASIL, 2011).

Entretanto, de acordo com Oliveira (2007) o aumento das coberturas de acesso na atenção básica, que em grande parte se deve pela implementação da ESF nos municípios, não significou, na mesma medida, um aumento de resolutividade dos problemas de saúde em todos os níveis de atenção. Uma hipótese que justifica isso é que há dificuldades a serem enfrentadas pelos profissionais para coordenar suas funções de trabalho. Organizar-se como equipe, conseguir cumprir atividades planejadas, discussões de casos clínicos, entre outros, que estão sendo escanteadas em relação ao cumprimento de forma mecânica das demandas espontâneas nas Unidades Básicas de Saúde, o que leva o serviço a uma maior burocratização e mais médico- centrado. (OLIVEIRA, 2007).

Assim, com o intuito de os profissionais de saúde atuarem e observarem a clínica para além de pedaços fragmentados foi criada a clínica ampliada (BRASIL, 2007), uma visão que vai além de apenas a doença do paciente, analisando também o sujeito como algo singular para que seja realizada a terapêutica mais adequada para aquele paciente como um todo, incluindo seu estilo de vida, suas relações interpessoais e suas problemáticas individuais. 
Dessa maneira, o Projeto Terapêutico Singular (PTS) surge como uma das ferramentas da clínica ampliada para que haja um cuidado integrativo. Ele reúne um conjunto de proposições de condutas terapêuticas para um ser em individual ou uma coletividade, advindo de uma discussão da equipe (BRASIL, 2007), a partir das dificuldades e potencialidades identificadas.

Para Pagani e Domitti (2007), o PTS aborda quatro fases: diagnóstico multiaxial, definição de metas, divisão de responsabilidades e reavaliação. Portanto, é uma ação interdisciplinar de saúde, em que são levadas em consideração todas as opiniões para auxiliar na compreensão do indivíduo que precisa de cuidado e para também ser possível a elaboração de propostas terapêuticas.

Destarte, a produção do PTS envolve uma relação profissional-usuáriofamília- comunidade que leva ao empoderamento e que sirva para consolidação do vínculo entre esses três agentes. De acordo com Silva et al (2016) essa produção pode usar como uma estratégia inicial a abordagem da contratualidade entre as pessoas, que seria dar a possibilidade desses atores serem reconhecidos como parceiros:

Por vezes, devido às inúmeras dificuldades vivenciadas, a pessoa chega à unidade de saúde com pouco poder contratual. Nesse momento, o profissional pode reconhecer que a pessoa está fragilizada e ter cuidado para preservá-la de maneira que sejam criadas possibilidades para que os contratos possam efetivamente ser cumpridos pela pessoa.

Espera-se que durante o manejo do contrato seja construída uma relação de reciprocidade, a qual requer do profissional paciência, tato e a capacidade de "estar com" o usuário. (SILVA et al., 2016, p. 02)

Portanto, observa-se que para a organização do PTS é essencial qualidade das conversas, com escuta ativa e troca de saberes, o que leva a confiança e vínculo entre o usuário e a equipe, sendo esse contato responsável pela compreensão da história do outro. E, é através disso que é possível um acompanhamento integralizado do usuário, que passa a ser visto de maneira mais abrangente. 
Dessa forma, o objetivo do trabalho é discutir o uso do PTS como ferramenta de construção de um cuidado integral, por meio da experiência em uma unidade de saúde da família.

\section{MATERIAIS E MÉTODOS}

O trabalho trata-se de um relato de experiência ocorrida na Unidade de Saúde da Família (USF) Mudança de Vida, localizada na cidade de João Pessoa (PB), por uma estudante de medicina do quarto período da Universidade Federal da Paraíba (UFPB), durante a disciplina "MHA 4 - Atenção à saúde da família I".

A disciplina é caracterizada pela sua articulação teórico-prática no âmbito da Assistência Básica em Saúde e deu-se no período de julho a novembro de 2018, sendo que a elaboração do PTS ocorreu de 31 de julho de 2018 a 2 de novembro de 2018. Ela tinha como objetivo a inserção do aluno na rede básica de saúde da cidade, e compreender a Estratégia Saúde da Família (ESF) enquanto proposta de reorientação da atenção básica e de organização do modelo tecno- assistencial a partir da unidade em que está inserido. Os estudantes permaneciam quatro horas semanais, nas terças-feiras pela manhã, na USF, assessorados pelo professor médico de família e pela comunidade, bem como mais duas horas semanais de discussão em sala de aula sobre as práticas, com formação teórica para realização das práticas.

Uma característica da disciplina é a utilização de tecnologias leves para a produção de saúde e desenvolvimento acadêmico do estudante, sendo elas o acolhimento, autonomização, criação de vínculo, entre outras. Dessa maneira, durante as práticas, através dessas estratégias, eram realizados atendimentos com os usuários, onde foi escolhido dentre eles um que apresentava vulnerabilidades, para construir o PTS de acordo com as necessidades identificadas junto à equipe. Assim, os estudantes selecionaram o caso da Senhora J para construir o PTS, por ela ser uma idosa que mora sozinha, com multimorbidades e que a primeira vista os estudantes e o preceptor perceberam que o PTS poderia ser uma estratégia de 
grande valia para minimizar as queixas apresentadas por ela. Por isso, para a realização desse relato de experiência foi selecionado esse caso.

A construção do PTS se deu a partir da realização de cinco encontros com a paciente, sendo alguns pelo atendimento na USF e outros por visita domiciliar a casa da usuária. Esse PTS inclui a história de vida da paciente, seu contexto familiar, o registro dos atendimentos, o plano de cuidado com metas a curto, médio e longo prazo e, por fim, as considerações finais do projeto. Além disso, como estratégia para a elaboração do plano de cuidado do PTS também foram utilizados instrumentos de coleta de dados familiares: o genograma e o ecomapa da paciente.

\section{RESULTADOS E DISCUSSÃO}

Foi selecionada para este trabalho a usuária Senhora J, de 86 anos. Uma mulher ativa, que mora sozinha no bairro Colinas do Sul, em João Pessoa (PB). É aposentada e sobrevive exclusivamente com o dinheiro da sua aposentadoria. Ela é hipertensa controlada, possui insuficiência cardíaca congestiva (ICC) e insuficiência renal crônica (IRC) - descoberta durante a elaboração do PTS - apresentando cansaço aos médios esforços decorrentes da ICC e dificuldade de locomoção. A paciente foi escolhida através de um atendimento realizado pelos estudantes com ela na USF, onde foi observado suas vulnerabilidades e notou-se que o PTS seria uma boa estratégia de cuidado para ela.

Sem parentes próximos vivos, solteira e sem filhos, ela cuida sozinha da casa e conta com o auxílio esporádico da vizinha para realização de tarefas mais complexas. Além disso, utilizava vários medicamentos (polifarmácia), não seguia uma dieta alimentar e reclamava da solidão que sentia e de que queria ter mais afazeres para preencher o seu muito tempo livre.

Foram realizados, como descrito na metodologia, atendimentos semanais e visitas domiciliares a paciente por uma dupla de estudantes de medicina. Nesses momentos, havia conversas para entender melhor a vida e o contexto social da 
paciente e a cada encontro era realizado a anamnese no modelo SOAP (registro de atendimento orientado por problemas).

Ademais, houve a criação de genograma, no qual é a representação gráfica e visual da família, suas relações e doenças apresentadas, o que auxiliou para um maior entendimento da queixa de solidão da paciente, pois foi possível representar visualmente a sua árvore genealógica e saber quem são os membros da sua família. Ao realizar isso, notou-se que a usuária não tem parentes vivos próximos a ela (na mesma cidade e estado), assim foi criado também um ecomapa, instrumento gráfico que ajuda na visualização das relações sociais apresentadas pela Sra. J, mostrando o grau de intensidade de cada relação, o que auxiliou no acompanhamento dela e na solução para as suas queixas. Através disso tudo, foi possível a implementação das condutas terapêuticas baseadas no plano de cuidado do seu PTS.

A partir desses encontros, foi criado um vínculo entre a usuária e as estudantes, o que favoreceu no cuidado integrado dela. De acordo com Campos (1997) esse vínculo leva a um aumento da eficiência das ações em saúde, além de favorecer a participação da usuária na prestação do serviço. Isso também contribui para a formação de sujeitos autônomos, pois na construção do vínculo o paciente é reconhecido como sujeito, que fala, julga e deseja (SCHIMITH E LIMA, 2004).

Nessa perspectiva, a paciente foi colocada como protagonista do seu cuidado, os atendimentos foram baseados no acolhimento das suas demandas e em escuta ativa, que segundo Lopes e Silva (2004) é o engajamento no sentido de "estar com" com o objetivo de desenvolver uma aceitação compreensiva um do outro.

Com base em tudo isso, foi possível a elaboração de um plano de cuidado individualizado para a paciente. Dessa maneira, nota-se que as proposições dadas pelo PTS para esse plano de cuidado devem decorrer de debate coletivo do caso, através de várias visões, em incluso a da própria paciente (BRASIL, 2010). Assim, foram criadas terapêuticas articuladas, que tem como finalidade a melhoria dos sintomas e ampliação da rede social com o intuito de mudar o curso do adoecimento (MORORÓ, 2010).

Destarte, foi traçado encaminhamentos a curto, médio e longo prazo para a resolução das demandas apresentadas. Assim, em curto prazo foi proposto a 
revisão dos medicamentos utilizados, pois a paciente queixava-se da dificuldade que era a utilização de tantos medicamentos, logo, observou-se que a usuária estava utilizando mais de um remédio com o mesmo princípio ativo e outros que não possuíam bom sinergismo farmacológico, sendo diminuído assim a quantidade de medicamentos usados diariamente e alterado suas doses para obter uma maior eficácia.

Durante as primeiras consultas foi realizado o diagnóstico de IRC da paciente, sendo assim necessário em curto prazo orientá-la sobre essa patologia, explicar que por ser uma doença crônica a senhora terá que lidar com ela para o resto da sua vida. Foi também informado a usuária como é o tratamento dessa doença, as restrições que ela causa e o que pode ser feito para prevenir piora do quadro.

Além disso, foi proposto em médio e longo prazo um plano alimentar para paciente nefropata e hipertenso, pois a mesma relatava e era notado que a paciente não seguia uma dieta adequada para as suas condições de saúde. É importante ressaltar, que esse plano foi baseado em alimentos regionais, de fácil acesso e de baixo custo, pois a paciente relatou ter dificuldade de seguir dietas anteriores por não conseguir comprar os ingredientes.

Também foi trabalhado com ela sobre os impactos das suas patologias na vida de um idoso e, por fim, foi abordada com a paciente a questão de como lidar com a solidão, pois ela morava só, não possuía parentes vivos próximos, tinha como única grande amiga a vizinha e possui dificuldades para se locomover para locais mais distantes visitar conhecidos. A partir dessa demanda foi proposta em longo prazo a realização de atividades de lazer, que ocupassem não só o tempo da paciente mais que gerassem uma forma de satisfação cotidiana, além de ser uma oportunidade de fazer novas amizades.

Dessa maneira, as principais vulnerabilidades da paciente foram minimizadas, as queixas apresentadas por ela na primeira consulta foram solucionadas e suas patologias crônicas controladas, o que possibilitou uma melhoria na sua qualidade de vida. Além disso, outro resultado obtido foi um estreitamento do vínculo da usuária com a unidade de saúde, que ao final do semestre relatou para as estudantes que possuía um grande apreço por elas e por toda equipe. Havendo, 
portanto o fortalecimento da participação da USF na rede de apoio dela, o que é de extrema importância no acompanhamento futuro do seu cuidado.

Assim, observou-se que o PTS vai além de um plano padrão que segue um modelo já definido, formado por profissionais que atuam individualmente e sem união, mas na verdade é uma coleção de proposições terapêuticas pensadas para um indivíduo, grupo ou família que possui que possui singularidades e individualidades, que são diferentes das de outras pessoas (BOCCARDO et al., 2011).

Portanto, para a construção do PTS foi necessário entender a situação de vida do paciente sobre a sua óptica e a de todos os membros da equipe, individualmente, levantar hipóteses diagnósticas que contemplem os aspectos orgânicos, subjetivos e sociais, os riscos e as vulnerabilidades, os limites e as potencialidades dos sujeitos (BRASIL, 2011).

Por fim, o PTS foi útil também para o aprendizado dos estudantes que o desenvolveram, possibilitando assim, um enriquecimento teórico-prático de grande valia para a formação tanto profissional quanto pessoal deles.

\section{CONSIDERAÇÕES FINAIS}

O PTS se apresentou como uma estratégia eficaz para a minimização das vulnerabilidades da paciente. Tendo sido possível a resolução das queixas apontadas pela paciente no decorrer das consultas e controle de suas patologias crônicas. Além disso, foi observada melhoras não só no contexto biológico, mas também no psicossocial, pois a usuária passou a ter a unidade como parte da sua rede de apoio e nela pessoas que ela gosta e aprecia socialmente.

Portanto, essa ferramenta se mostrou de grande utilidade no cuidado a paciente do relato de experiência, pois trouxe uma visão integrada dela que vai além da tradicional visão biomédica hospitalocêntrica, focada apenas na doença e seu tratamento medicamentoso, e sim englobando os aspectos biopsicossociais inerentes de todo ser humano e mostrando que o cuidado em saúde é algo dinâmico 
e que deve ser individualizado de acordo com as necessidades de cada usuário da comunidade.

Vale ressaltar também, que foi uma importante fonte de aprendizado para os estudantes que participaram da elaboração desse projeto, pois através desse contato conhecer novas formas de fazer saúde, levando a um grande incremento no ensino deles e possibilitando um grande avanço nas suas formações acadêmicas e pessoais.

Além disso, a implantação do PTS vem motivar a reavaliação do modelo biomédico hospitalocêntrico, com a superação do pensar profissional individualizado e apenas com fim prescritivo, cuja única fonte é o saber científico em detrimento do reconhecimento do saber do paciente. Devendo, portanto, haver mais debates, reflexões e problematizações acerca do assunto por parte de todos os integrantes do sistema de saúde, para que, dessa forma, seja possível cada vez mais, melhorar a assistência à saúde no Brasil. 


\section{REFERÊNCIAS BIBLIOGRÁFICAS}

BOCCARDO, A. C. S. et al. O projeto terapêutico singular como estratégia de organização do cuidado nos serviços de saúde mental. Rev. Ter. Ocup. Univ. São Paulo, v. 22, n. 1, p. 85-92, jan./abr. 2011.

BRASIL. Ministério da Saúde. Secretaria de Atenção à Saúde. Núcleo Técnico da Política Nacional de Humanização. Clínica Ampliada, Equipe de Referência e Projeto Terapêutico Singular. 2. ed. Brasília: Ministério da Saúde, 2007.

BRASIL. Ministério da Saúde. Secretaria de Atenção à Saúde. Política Nacional de Humanização. Atenção Básica. Brasília: Ministério da Saúde, 2010.

BRASIL. Ministério da Saúde. Portaria n. 2.488, de 21 de outubro de 2011. Aprova a Política Nacional de Atenção Básica, estabelecendo a revisão de diretrizes e normas para a organização da Atenção Básica, para a Estratégia Saúde da Família (ESF) e o Programa de Agentes Comunitários de Saúde (PACS). Brasília: Ministério da Saúde, 2011.

CAMPOS, G.W.S. Considerações sobre a arte e a ciência da mudança: revolução das coisas e reforma das pessoas. O caso da saúde. In: Cecilio LCO, organizador. Inventando a mudança na saúde. $2^{\mathrm{a}}$ Ed. São Paulo: Editora Hucitec; 1997. p. 29-87.

LOPES, Marta Julia Marques; SILVA, João Luis Almeida da. Estratégias metodológicas de educação e assistência na atenção básica de saúde. Revista Latino-americana de Enfermagem, [s.i.], v. 12, n. 4, p.683-688, jul. 2004.

MINAYO, Maria Cecília de Souza. Os 20 anos do SUS e os avanços na vigilância e na proteção à saúde. Epidemiologia e Serviços de Saúde, [s.I.], v. 17, n. 4, p.245-246, dez. 2008. Instituto Evandro Chagas.

MORORÓ, M. E. M. L. Cartografias, desafios e potencialidades na construção de projeto terapêutico em Centro de Atenção Psicossocial. 2010. 110 f. Dissertação (Mestrado) Escola de Enfermagem da Universidade de São Paulo, Universidade de São Paulo, São Paulo, 2010.

OLIVEIRA, Gustavo Nunes de. O Projeto terapêutico como contribuição para a mudança das práticas de saúde. 2007. 176 f. Dissertação (Mestrado) - Faculdade de Ciências Médicas, Universidade Estadual de Campinas, Campinas, 2007.

PAGANI, Rosani; ANDRADE, Luiz Odorico Monteiro de. Preceptoria de território, novas práticas e saberes na estratégia de educação permanente em saúde da família: o estudo do caso de Sobral, CE. Saúde e Sociedade, [s.I.], v. 21, n. 1, p.94-106, maio 2012. FapUNIFESP (SciELO).

SCHIMITH, Maria Denise; LIMA, Maria Alice Dias da Silva. Acolhimento e vínculo em uma equipe do Programa Saúde da Família. Cadernos de Saúde Pública, Rio de Janeiro, v. 6, n. 20, p.1487-1494, nov-dez. 2004.

SILVA, Ariná Islaine da et al. Projeto terapêutico singular para profissionais da estratégia de saúde da família. Cogitare Enfermagem, [s.I.], v. 21, n. 3, p.01-08, 19 set. 2016. Universidade Federal do Paraná. 